\title{
Cancer genomic medicine: clinical validation and utility
}

\begin{abstract}
Rudimentary understanding of biological significances of various genomic alterations is causing slow paced translation of cancer genome sciences to personalized medicine. Comprehensive databases of genetic variations associated to effective cancer prevention approaches, early detection and treatment responses are required to successfully utilize the large amounts of information generated by current genetic tools. Efforts towards incorporation of advanced genomic technologies into clinical practice will help bringing research driven improvements in cancer diagnosis and individualized medical care.
\end{abstract}

Keywords: cancer genome, clinical utility, genomic technology, multi-molecular testing panels
Volume 3 Issue 3 - 2015

Nidhi Gupta

Centrillion Technologies, USA

Correspondence: Nidhi Gupta, Centrillion Technologies, 2500 Faber Place, Palo Alto, CA, USA, Tel 7| 8-406-5732, Email nidhi_in84@yahoo.com

Received: October 25, 2015 | Published: October 29, 2015
Abbreviations: NGS, next generation sequencing; CLIA, clinical lab improvement amendments; CMS, centers for medicare \& medicaid services; CAP, college of American pathologist; ASCO, American society of clinical oncology; NCCN, National comprehensive cancer network; FDA, food and drug administration; $\mathrm{CDC}$, centers for disease control and prevention; FNIH, foundation for the National institutes of health

\section{Introduction}

Recent breath-taking developments in next generation sequencing (NGS) and microarray platforms have triggered a genomic revolution. Whole genome-wide associated studies have helped in establishing numerous genetic variations involved in various common diseases. Significant technical breakthroughs have revealed immense complexity of the cancer genome that exists at multiple levels including in analogous tumors in same individuals. Several commercially available molecular diagnostic panels for cancer are attempting to develop sufficient evidence to demonstrate their successful clinical validation and utility. To provide patients customized assessment of potential risks of developing several types of cancers because of genetic predisposition and preventive measures tailored to their requirements, the physicians and genetic counselors at cancer genetics clinics all around the country are using rapidly evolving genomic technologies for identification of genetic variants contributing to various types of cancers.

\section{Molecular marker panels and testing}

Besides microarray and sequencing, several other techniques are being used for genomic and molecular profiling of cancer. ${ }^{1}$ Traditionally, pharmacogenomic applications in health centers rely on single cancer genomic marker identification to direct targeted therapy and treatment decisions for various types of cancers. ${ }^{2}$ Several single molecular markers have been well established for their sensitivity or resistance to specific drugs (Table 1). But with advances in technologies and declined costs of NGS, single gene testing has advanced into multi-gene panels to identify larger number of variations driving tumorigenesis. Several tumor panels (for example Foundation One by Foundation Medicine and SuraSeq by AsuraGen), accredited by Clinical Lab Improvement Amendments (CLIA, regulated by Centers for Medicare \& Medicaid Services (CMS) for laboratory testing) and College of American Pathologist (CAP), are now commercially available to identify variable number of cancer genome markers. ${ }^{3}$

Table I Examples of single cancer genomic marker associated with pharmacogenomic applications

\begin{tabular}{|c|c|c|c|}
\hline \multirow{2}{*}{$\begin{array}{l}\text { Genomic } \\
\text { marker }\end{array}$} & \multicolumn{2}{|l|}{ Associated drug (s) response } & \multirow{2}{*}{ Tumor site } \\
\hline & Increased resistance & Increased sensitivity & \\
\hline BRCAI & Cisplatin, GW44I 756, ABT-263, JNKInhibitor VIII & AMG-706,ABT-888,AZD-228I, Erlotinib & Breast and Ovary \\
\hline ALK & Roscovitine & $\begin{array}{l}\text { Vorinostat, AZD-228I, Methotrexate, CEP-70I, ZM- } \\
447439, \mathrm{SBI} 6763\end{array}$ & Lung \\
\hline MYC & AZD6482 & $\begin{array}{l}\text { Vinblastine, Cisplatin, GDC-0449,Vinorelbine, Bleomycin, } \\
\text { Embelin,Thapsigargin }\end{array}$ & Breast \\
\hline KRAS & $\begin{array}{l}\text { Vorinostat, NVP-BEZ235, AZD8055, PD- } 173074 \text {, } \\
\text { Axitinib, AZD6482BX-795, GDC094I }\end{array}$ & $\begin{array}{l}\text { AZ628, PD-032590 I, Cl-1040, RDEAI I9, AZD6244, } \\
\text { BMS-754807,TW37 }\end{array}$ & Colon \\
\hline BRAF & AZD6482, Axitinib, GDC094I, BIBW2992, & $\begin{array}{l}\text { SB590885, PLX4720, RDEA I I 9, PD-032590 I, Cl-1040, } \\
\text { AZ628, AZD6244, CHIR-9902 I, Docetaxel, Obatoclax } \\
\text { Mesylate, FH535, Embelin, AICAR }\end{array}$ & Melanoma \\
\hline APC & $\begin{array}{l}\text { Temsirolimus, DMOG,Vinblastine, NVP-BEZ235, } \\
\text { Pazopanib, AZD6482, JNJ-26854I 65, ATRA, BX- } \\
\text { 795, NU-744I, GDC094I, Elesclomol, Cisplatin }\end{array}$ & BMS-754807, PD-032590 I, RDEA I I9, NSC-87877, & Colon \\
\hline
\end{tabular}


Although these tests and panels are highly reliable, several factors, such as methods of collection and handling of tissue samples for different assays, can contribute to variations and affect the uniformity in the results from different techniques or panels. Such variations can be reduced by preparation of the samples in a specific standardized procedure for different assays. This might increase the precision and accuracy of data collected from different assays and help in building confidence in results, analytical validation, variant calling and data interpretation by clinicians. Computational algorithms should be developed for therapeutic purposes to handle large amounts of data, and integrate interpretive and decision making processes.

Several databases summarize the polymorphisms, variations and mutations found in cancer genome and associated potential drug response or clinical action. ${ }^{4,5}$ These databases are used by physicians and cancer genome specialists to study and interpret data acquired from various commercially available tests and panels.
In another hypothesis, rather than involvement of an individual variation or marker in cancer treatment response, collective role of multiple molecular markers on specific cellular pathways, such as proliferation, metabolism, DNA repair or apoptosis, is proposed to guide the cancer treatment (Table 2). ${ }^{1}$ According to it, measurement of a large number of relative genetic and other molecular markers in cancer tissue may help to identify the biological pathways involved in specific cancer types. Such information could aid in selection of a certain treatment based on characteristics and stage of the disease. In such cases genomic and molecular variations or markers will required to be categorized and established for involvement in specific mechanistic pathways that affect cancer behavior and treatment. Furthermore, development of commercial tests and panels, and demonstration of their analytical and clinical validation before commercialization for clinical purposes will be required to identify the multiple markers or, more specifically, the pathways affected in specific tumor sample in order to strategize a personalized treatment for a patient.

Table 2 Examples of multi-molecular markers associated with specific cancer types

\begin{tabular}{lll}
\hline Cancer types & Associated markers & guidelines and recommendations \\
\hline Colon & RAS mutation (KRAS, NRAS), BRAF & NCCN,ASCO \\
Non-Small-Cell Lung Cancer & EGFR, ALK/ROSI, KRAS & NCCN \\
Metastatic Melanoma & BRAF v600 & NCCN \\
Breast & ESRI/ MYC/ BRCAI & NCCN,ASCO \\
\hline
\end{tabular}

NCCN, National comprehensive cancer network

ASCO,American society of clinical oncologyx

\section{Challenges in clinical validity}

The main objective of cancer genomic medicine is to establish the biological relevance of cancer genomic profiles and application of these genetic tools to investigations of cancer prevention strategies and variable treatment responses. Lack of clear uniform recommendations at various levels from laboratory to public health is the major barrier in instituting the clinical utility of cancer genomic studies. Development of practical guidelines for evaluation of analytical validation, assay performance in lab settings, and clinical validation, association between test results and pathophysiological status of cancer is still under progress.

Ability to validate functionally relevant cancer driver variations is hindered by several limitations such as experiment models, assay conditions, source of data originated and accuracy of information generated from the various tests. Variations can be introduced in experimental systems by cell type, cancer developmental stage, and microenvironment and sample collection methods. To address these challenges, understanding about contribution of each tumor driver event towards origin and maintenance of cancer and conversion of genomic outcome into medical action is required.

Poor standardization of different test assays for analysis of same samples results into method-specific issues. Different methods of sample preparation for different assays, use of different lab procedures, treatment reagents and computational analysis approaches adds more levels of disparities. Clinical utility depends on accuracy and specificity of diagnostic data. Several organizations such as American Society of Clinical Oncology (ASCO) and CAP are generating guidelines for standardization of these methods and assays.

With novel genomic technologies, huge amounts of raw data are produced which needs to be interpreted correctly. Generally for appropriate illustration and clinical utility of these data, specific parameters are applied to filter the generated information. Lack of comprehensive understanding of disease parameters and clinical settings while applying such filtering constraints to raw data can lead to mis-interpretation of acquired raw data. Guidelines for regulation of data filtration and classification are still required to avoid discrepancies in validation and utility.

To meet the aim of translation of cancer genome into patient customized therapy, understanding of underlying mechanisms by which genetic alterations influence phenotypic characteristics of cancer is required. Despite the availability of more comprehensive representation of cancer genomic landscape, there is lack of consensus to categorize variations as relative or non-significant for medical purposes. Although early cancer genomic studies have provided insights into the role of genetic variations in driving diagnostic and therapeutic approaches for cancer treatment, standardized and widely accepted guidelines are still required to catalogue newly identified variations as clinically important or actionable.

At first, there is lack of clear evidence that use of commercially available assays will lead to improved patient outcomes than other solutions. In addition to this, inadequate designs of clinical trials and infrastructure, to sustain the implementation of assays results, are another hurdle in successful clinical usage of molecular diagnostic tests. Several ongoing clinical trials are utilizing pre-marketed or commercially available panels and tests. Major objective of these trials is to determine time required to acquire molecular profiling results, accuracy and specificity of outcomes, fraction of successful tests among attempted, percentage ability to implement results of different types of tests and more. Various factors govern the success of these trials such as number of screened participants, informed consents and awareness of patients, appropriate pre-test genetic counseling 
and regular follow-up, random or non-random trial evaluations and others. Studies of genetic variation in tumor tissue only may also cause misguided treatment strategies. Although large number of clinical trials are ongoing to study tumor progression free survival in participants to determine longer term advantages of treatment strategies used based on molecular diagnostic tests, most of the tests and panels being used for identification of genomic alterations and treatment plans are non-Centers for Disease Control and Prevention (CDC) and the U.S. Food and Drug Administration (FDA) approved and still require standardization. Recently some tests and panels such as CancerTypeID, miReview and PathworkDx have failed to provide sufficient evidence for their clinical importance. ${ }^{6}$ Post-market commercial studies and longer term clinical use of cancer diagnostic tests can provide more evidence towards generation of standard guidelines, but these can be expensive, time consuming and require defined large populations for studies.

Clinical trials are also required to be diversified and analyzed separately based on different markers, cancer or treatments. Studies combining these clinical trials based data may raise the issues in uniformity and implementation of treatment strategies.

In most cases cancer genomic profiles may help the patients to reduce the potential risk of developing cancer; for example patients with genetic pre-disposition to lung cancer are suggested to stay in smoke free environment, ${ }^{7}$ but another critical question to implication of genetic variability and expression profiles is variable expression of disease in different environmental exposures.

In certain cases such genetic testing may present medically useless information that could not help in predication of prevention or treatment strategies of disease which raises ethical issues regarding screening and genetic testing. ${ }^{8}$

\section{Current prospective and practices}

Several public and private agencies such as FDA, National Institutes of Health (NIH)/ National Cancer Institute (NCI) and clinical researchers are working in synchronization to provide guidelines and practical definitions of clinical utility of cancer diagnostic tests to developers. Foundation for the National Institutes of Health (FNIH) Biomarkers consortium was developed to catalogue qualification of recognized alterations as biomarkers for diagnosis and treatment of cancer. ${ }^{6}$ Center for Medical Technology (CMTP) has outlined an Effectiveness Guidance Document identifying gaps and containing references to improve the evidence base for development and clinical utility of molecular diagnostics. ${ }^{9} \mathrm{NCI}$ and CMTP are also recommending the developers to clearly define the anticipated use and clinical perspective of commercial products under development at early stages. Studies of these products in intended populations may assure the accuracy of performance and results. Considerations of advantages of new developed tests over pre-existing ones such as accessibility, expenses, convenience and accuracy are also recommended. Another concept added in list of features for development of new test is 'fit for purpose' that allows inadequate performance characteristics (specificity or sensitivity) of the test in certain applications but fills a gap in medical needs such as identification of small population that elucidates high therapeutic efficacy.

Although pre-market demonstration of clinical utility of newly developed diagnostic tests is still un-regulated, currently commercialization of such products is controlled by FDA or CMS which requires evaluation of analytical validity of these tests. FDA and CMS is also encouraging the developers to create project plans that include assay testing and implementation timeline for clinical utility, carefully designed clinical trials, analysis and total costs.

\section{Conclusion}

Application of rapidly emerging genomic technologies to identify clinically actionable genetic variants requires more regulated studies and evaluation. Currently no universally accepted standards and references are available for implementation of cancer genome data acquired by various methods. New discoveries suggesting more genetic variations related to different types of cancer are required to be determined as driver or passenger mutations and categorized appropriately if influential on cancer treatment approaches and drug responses. Clinical trials to demonstrate the utility of cancer genome profiles developed by various techniques and to establish the relationship between genomic variations and variable effects on a population should be carefully designed and studied.

Additional contributions of various public and private sources would allow the refined representation of clinical significance of cancer genome associated studies.

\section{Acknowledgments}

None.

\section{Conflicts of interest}

The authors declare there is no conflict of interests.

\section{Funding}

None.

\section{References}

1. BlueCross BlueShield Association. Special report: multiple molecular testing of cancers to identify targeted therapies. Technol Eval Cent Assess Program Exec Summ. 2013;28(1):1-2.

2. NIH. Bringing the genomic revolution to the public. National Human Genome Research Institute; 2012.

3. Marrone M, Filipski KK, Gillanders EM, et al. Multi-marker solid tumor panels using next-generation sequencing to direct molecularly targeted therapies. PLoS Curr. 2014.

4. Catalogue of somatic mutaions in cancer. COSMIC.

5. Variation Viewer. NCBI.

6. Parkinson DR1, McCormack RT, Keating SM, et al. Evidence of clinical utility: an unmet need in molecular diagnostics for patients with cancer. Clin Cancer Res. 2014;20(6):1428-1444.

7. Journal of The American Medical Association. Genetic predisposition can play an important role in development of lung cancer. ScienceDaily. 2005

8. Hayden EC. Alzheimer's tests under fire. Nature. 2008;455(7217):1155.

9. Deverka P, Messner D, Dutta T. Effectiveness guidance document: evaluation of clinical validity and clinical utility of actionable molecular diagnostic tests in adult oncology. Center for medical technology policy (CMTP); 2013:1-58. 